\title{
A rule-based classifier with accurate and fast rule term induction for continuous attributes
}

Conference or Workshop Item

Accepted Version

Almutairi, M., Stahl, F. and Bramer, M. (2019) A rule-based classifier with accurate and fast rule term induction for continuous attributes. In: 17th International Conference on Machine Learning and Applications, 17th to 20th of December 2018, Orlando, Florida, pp. 413-420. Available at https://centaur.reading.ac.uk/80938/

It is advisable to refer to the publisher's version if you intend to cite from the work. See Guidance on citing.

Published version at: https://ieeexplore.ieee.org/document/8614093

All outputs in CentAUR are protected by Intellectual Property Rights law, including copyright law. Copyright and IPR is retained by the creators or other copyright holders. Terms and conditions for use of this material are defined in the End User Agreement.

www.reading.ac.uk/centaur 
Central Archive at the University of Reading

Reading's research outputs online 


\section{A Rule-Based Classifier with Accurate and Fast Rule Term Induction for Continuous Attributes}

\author{
$1^{\text {st }}$ Manal Almutairi, Frederic Stahl \\ Department of Computer Science \\ University of Reading \\ Reading, United Kingdom \\ Email: Manal.Almutairi@pgr.reading.ac.uk \\ F.T.Stahl@ reading.ac.uk
}

\author{
$2^{\text {nd }}$ Max Bramer \\ School of Computing \\ University of Portsmouth \\ Portsmouth, United Kingdom \\ Max.Bramer@port.ac.uk
}

\begin{abstract}
Rule-based classifiers are considered more expressive, human readable and less prone to over-fitting compared with decision trees, especially when there is noise in the data. Furthermore, rule-based classifiers do not suffer from the replicated subtree problem as classifiers induced by top down induction of decision trees (also known as 'Divide and Conquer'). This research explores some recent developments of a family of rulebased classifiers, the Prism family and more particular G-PrismFB and G-Prism-DB algorithms, in terms of local discretisation methods used to induce rule terms for continuous data. The paper then proposes a new algorithm of the Prism family based on a combination of Gauss Probability Density Distribution (GPDD), InterQuartile Range (IQR) and data transformation methods. This new rule-based algorithm, termed G-Rules-IQR, is evaluated empirically and outperforms other members of the Prism family in execution time, accuracy and tentative accuracy.

Index Terms-Modular Classification Rule Induction, Dynamic Rule Term Boundaries, Interquartile Range Rule Term Boundaries
\end{abstract}

\section{INTRODUCTION}

Decision tree based classifiers are popular for their accuracy and their simplicity to be converted into a set of rules by transforming each leaf of the tree into a rule. However, when dealing with large datasets, decision trees tend to become very large, complex, and difficult to understand. Consequently, the rules constructed through 'Divide and Conquer' strategy and extracted from the resulting decision tree inherit the tree's complexity and thus may have unnecessary repeated tests which can lead to redundant rulesets [1], [2]. In this context, research has been carried out aiming to overcome or reduce these problems and to produce a simple reliable ruleset using pruning methods. Among others, C4.5rules [3], [4] and CART [2] are examples of such algorithms. Nevertheless, [5]-[7] argue that there is no single study which adequately achieves this goal. Cendrowska [6] recognised the disadvantages of generating a ruleset in the form of a decision tree and criticises this method as a main source of over-fitting due to the replicated sub-tree problem that occurs when rules with no common attributes are forced to fit in a tree structure. Therefore, she developed Prism as an alternative expressive rule induction algorithm that follows a different rule induction approach called 'Separate and Conquer'. This approach can extract ifthen rulesets directly from training data. Several experiments conducted in [8] indicate that Prism is an ideal representative for 'Separate and Conquer' algorithms, it cannot only perform at the same level of accuracy as tree based methods but also in most cases outperforms them in terms of classification accuracy and computational efficiency, especially if there is noise in the data. RIPPER [9] and CN2 [10] are also examples 'Separate and Conquer' classifiers. However, Prism was the first algorithm that used top-down search without being controlled by a particular randomly selected pair of positive and negative instances which makes the algorithm more stable [11]. Despite that, as it can be seen in Algorithm 1, Cendrowska's original Prism is unable to handle continuous attributes and hence it requires converting them into categorical attributes prior to training stage. For the purpose of improving the computational efficiency of Prism, modified and also parallel versions of Prism have been developed in recent studies [7], [8], [12] motivating the use of Prism in this research. Previous work [13], [14] proposed new rule term structures for continuous attributes based on Gaussian Probability Density Distribution (GPDD); termed G-Prism-FB and G-Prism-DB. Section II provides further details of these continuous rule term induction approaches and their limitations. This paper proposes a new approach for inducing rule terms from continuous attributes for Prism based on quartiles and Interquartile Range termed G-Rules-IQR. This new version has an improved accuracy and lower computational costs compared with previous Prism versions. Also, we incorporated a method in G-Rules-IQR to resolve the normally distributed data assumption drawbacks that G-Prism-FB and G-Prism-DB suffer from.

This paper is organised as follows, Section II discusses related work on the Prism family of algorithms and Section III introduces and explains the in this paper proposed G-RulesIQR algorithm. Section IV provides an exhaustive empirical evaluation of the new algorithms compared with competing members of the Prism family of algorithms. This is followed by concluding remarks in Section V.

\section{Related Work: The Prism Family of Algorithms FOR INDUCING MODUlaR CLASSIFICATION RULES}

As shown in Algorithm 1, Prism uses a conditional probability theory to induce a rule term that covers the selected class $C$ 
in the training dataset $D$. All the examples that do not belong to $C$ are discarded. Prism continues to build the rule $R$ and at each step, tries to generate the perfect rule i.e. rules that cover training instances with a $100 \%$ accuracy of the current subset of the training data. The algorithm always resets training data to its original state before repeating the process and inducing more rules for the next target class. Prism involves five nested loops and hence building rulesets from high dimensional and large sample sizes is computationally expensive.

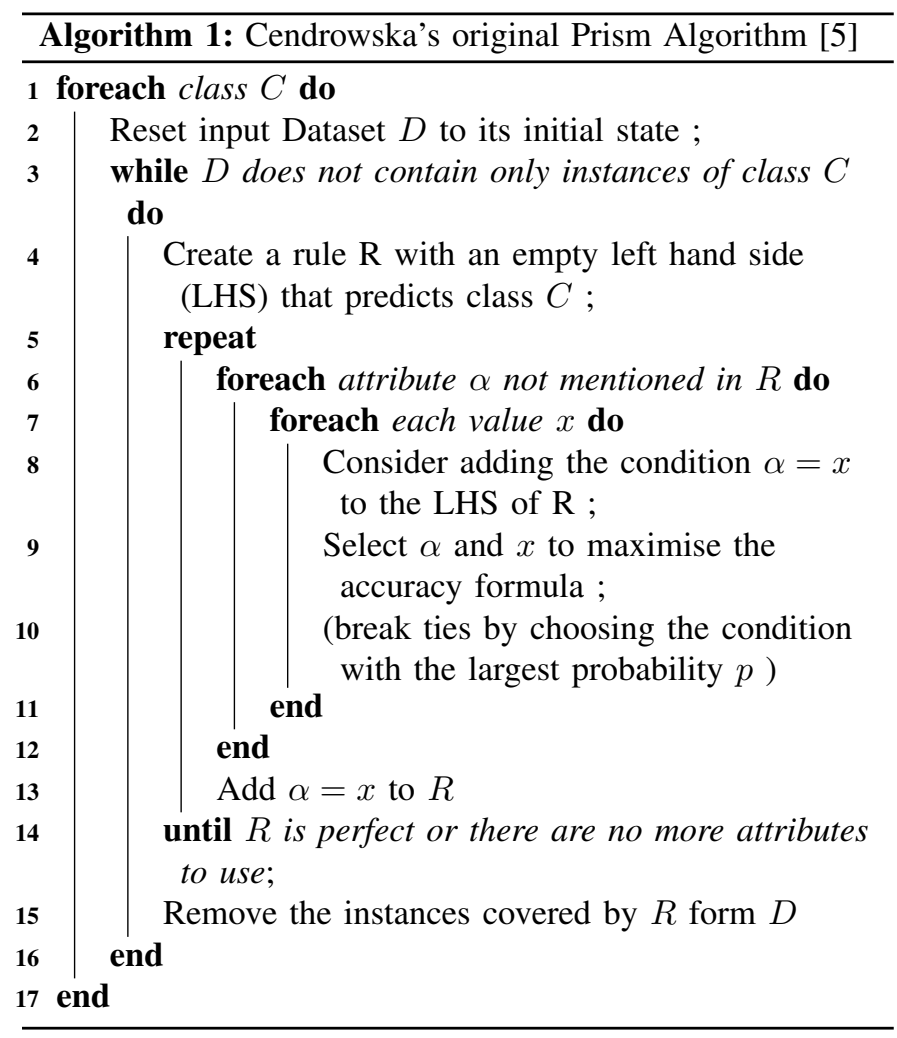

The original development of the Prism algorithm triggered several studies aiming to improve its performance, which are termed collectively the Prism family of algorithms. The first variation of Prism was described in [15] in order to overcome the limitation of original Prism which can only train from categorical attributes. This extended version of Prism is illustrated in Algorithm 2. It uses a local binary discretisation method called cut-points calculations (also referred to as binary splitting) to deal with a continuous attribute $\alpha$ by discretising continuous attribute values $v$ through rule terms of the form $(\alpha \leq v)$ and $(\alpha>v)$. This local discretisation is computationally very expensive and hence causes long training times. Variations of Prism have been developed to speed up the algorithm. As such PrismTCS [12] does not reset the dataset to its original size for each class label by removing the outer loop of the algorithm and introducing an order in which rules are induced. Another member in the Prism family called PMCRI [7] is a parallel and thus more scalable version of PrismTCS.

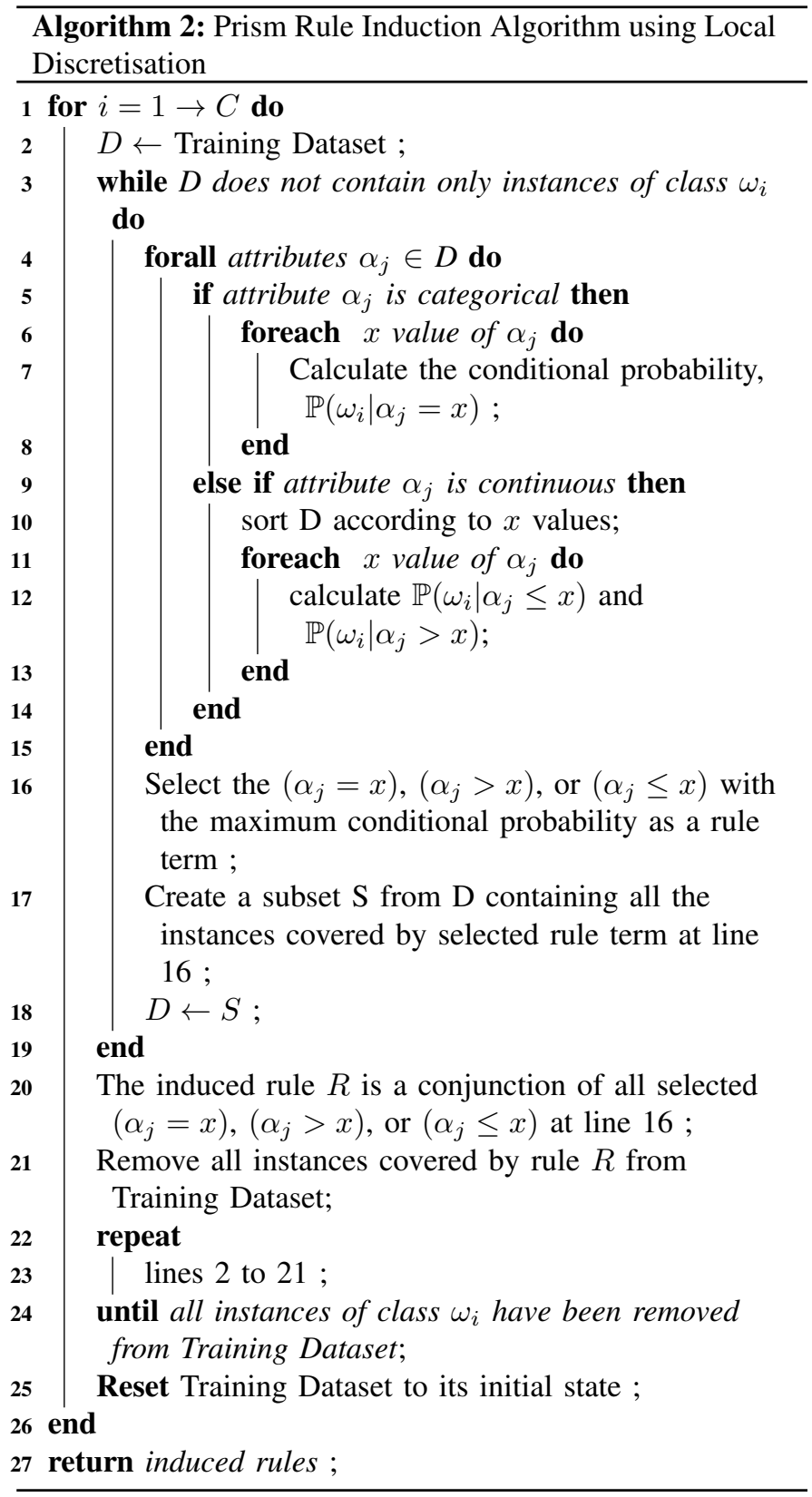

A. G-Prism with Fixed Boundaries (G-Prism-FB) and $G$ Prism with Dynamic Boundaries (G-Prism-DB)

The authors of [13] introduced two new Prism family members based on a new rule term structure that makes use of GPDD to induce computationally efficient continuous rule terms and to improve the classification performance of Prism based classifiers. These were termed G-Prism-FB and G-Prism-DB where G stands for GPDD, FB and DB refer to the type of rule term boundaries either fixed or dynamic. The new rule term structure is loosely based on the rule term structure used in [16], which presented a classifier for real-time streaming data. The main advantages of this rule induction approach is that the generated rule terms are more expressive and computationally less demanding compared with 
(a)

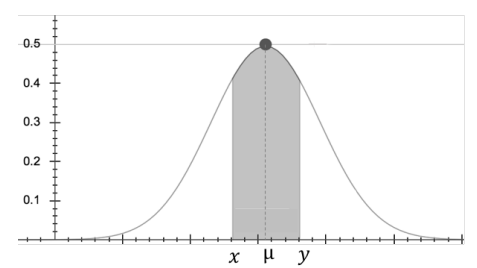

Fig. 1. The shaded area represents a range of values of attributes $\alpha_{j}$ for class $\omega_{i}$. (a) $68 \%$ of all possible values, (b)

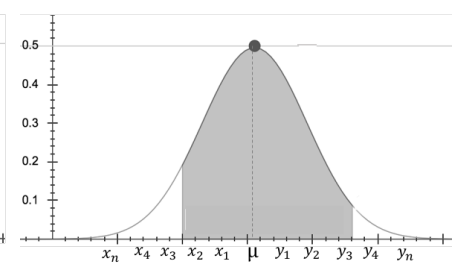

(b) $95 \%$ of all possible (b) $95 \%$ of all possible values the binary splitting approach where two candidate rule terms have to be generated for every possible attribute value (cutpoint calculations) before selecting the rule term with highest conditional probability (with which it covers the target class) to be appended to the rule currently being learned. As shown in Figure 1(a), G-Prism-FB can produce a rule term in the form of $(x<\alpha<y)$ by using a class conditional density probability of the Gaussian distribution function, where $x$ and $y$ are valid continuous attribute values. Like in [16] $x$ and $y$ are set to the next closest values left and right of the $\mu$ of the attribute's values. This algorithm resulted in a better accuracy compared with original Prism that uses binary splitting with cut-point calculations to deal with a continuous attribute [13]. However, the setting of $x$ and $y$ does not explore the rule term boundaries and in fact the best rule term boundaries may lie further left and right of $\mu$ than just the next attribute values. Thus GPrism-FB has been expanded to cover a user defined maximum number of values left and right of $\mu$. Let $k$ be the number of user defined values left and right of $\mu$ to be considered. The larger $k$ the more possible rule terms will be evaluated per attribute, such as $\left(x_{1}<\alpha \leq y_{3}\right),\left(x_{3}<\alpha \leq y_{5}\right)$, $\left(x_{2}<\alpha \leq y_{4}\right), \ldots\left(x_{n}<\alpha \leq y_{k}\right)$ as illustrated in Figure 1(b). This algorithm was termed G-Prism-DB. However, the larger $k$ the more computation is required as more rule term candidates have to be evaluated.

\section{B. Evaluation Summary of G-Prism-FB, G-Prism-DB and Prism}

Both G-Prism approaches (Fixed and Dynamic) and their predecessor Prism have been evaluated empirically and comparatively in [13] using 6 metrics and 11 datasets.

Summary of Results:

Overall, G-Prism-DB achieved a marginally better classification accuracy compared with G-Prism-FB and Prism using binary splitting except for the number of rules and abstaining rate where Prism with binary splitting performs better. The abstaining rate can be linked to the lower coverage of data instances per rule term in G-Prism in general. However, GPrism-DB generated marginally fewer rules and has a lower abstaining rate compared with G-Prism-FB.

\section{Limitations:}

Please note that all here listed limitations, except limitation 5 (which is subject to future work), will be revisited in Section III which describes the proposed G-Rules-IQR algorithm.
1) Execution time: Prism and G-Prism-DB are more computationally expensive than G-Prism-FB as a result of frequent cut-points calculations for Prism and multiple rule term bounds evaluation for G-Prism-DB. However, as expected, both approaches of G-Prism are faster than original Prism. Please note that this evaluation metric has not been used in [13], however, it is covered in Section IV in this paper.

2) Abstaining Rate: the abstaining rate for both approaches of G-Prism is higher than the one of original Prism. This will decrease the accuracy of the classifiers despite the higher tentative accuracy values. This is because abstained instances were counted as misclassifications.

3) User defined threshold: the user has to define the maximum number rule term boundary values to the left and right of $\mu$ (by default six values to the left and right). However, the optimal boundary may lie beyond this user defined value and is dependent on the number of training instances. This is because the larger the number of training instances the more likely it is that there are more distinct values. Thus the larger the number of distinct values, the more likely it is that the maximum boundary is closer to $\mu$.

4) Normal Distribution Assumption: in [13], the authors did not test the attributes' distributions in the experiments, yet the evaluation results reflect a good performance for G-Prism-DB in most cases. However, it is still possible that G-Prism algorithms may not perform as well on attributes that are not normally distributed due to the use of GPDD.

5) Attribute Dependencies: G-Prism algorithms do not take potential dependencies between attributes into account to further improve rule quality and expressiveness.

Limitations 1 to 4 are addressed in the research presented in this paper.

\section{G-RULES-IQR ALGORITHM}

The G-Rules-IQR approach is highlighted in Algorithm 3. The folowing subsections describe the new rule term induction procedure for continuous attributes and how the algorithm enables the induction of rule terms using GPDD, even if these attributes are not normally distributed.

\section{A. Using GPDD to Induce Rule Terms directly from Contin- uous Attributes}

The Gaussian distribution is calculated for each continuous attribute $\alpha_{j}$ with mean $\mu$ and variance $\sigma^{2}$ from all the values associated with classification $\omega_{i}$. The conditional probability for class $\omega_{i}$ is calculated using Equation 1.

$$
\mathbb{P}\left(\alpha_{j} \mid \omega_{i}\right)=\mathbb{P}\left(\alpha_{j} \mid \mu, \sigma^{2}\right)=\frac{1}{\sqrt{2 \pi \sigma^{2}}} \exp \left(-\frac{\left(\alpha_{j}-\mu\right)^{2}}{2 \sigma^{2}}\right)
$$

Hence, a value for $\mathbb{P}\left(\omega_{i} \mid \alpha_{j}\right)$ or equivalently $\log \left(\mathbb{P}\left(\omega_{i} \mid \alpha_{j}\right)\right)$ can be calculated as shown in Equation 2. This value is then 


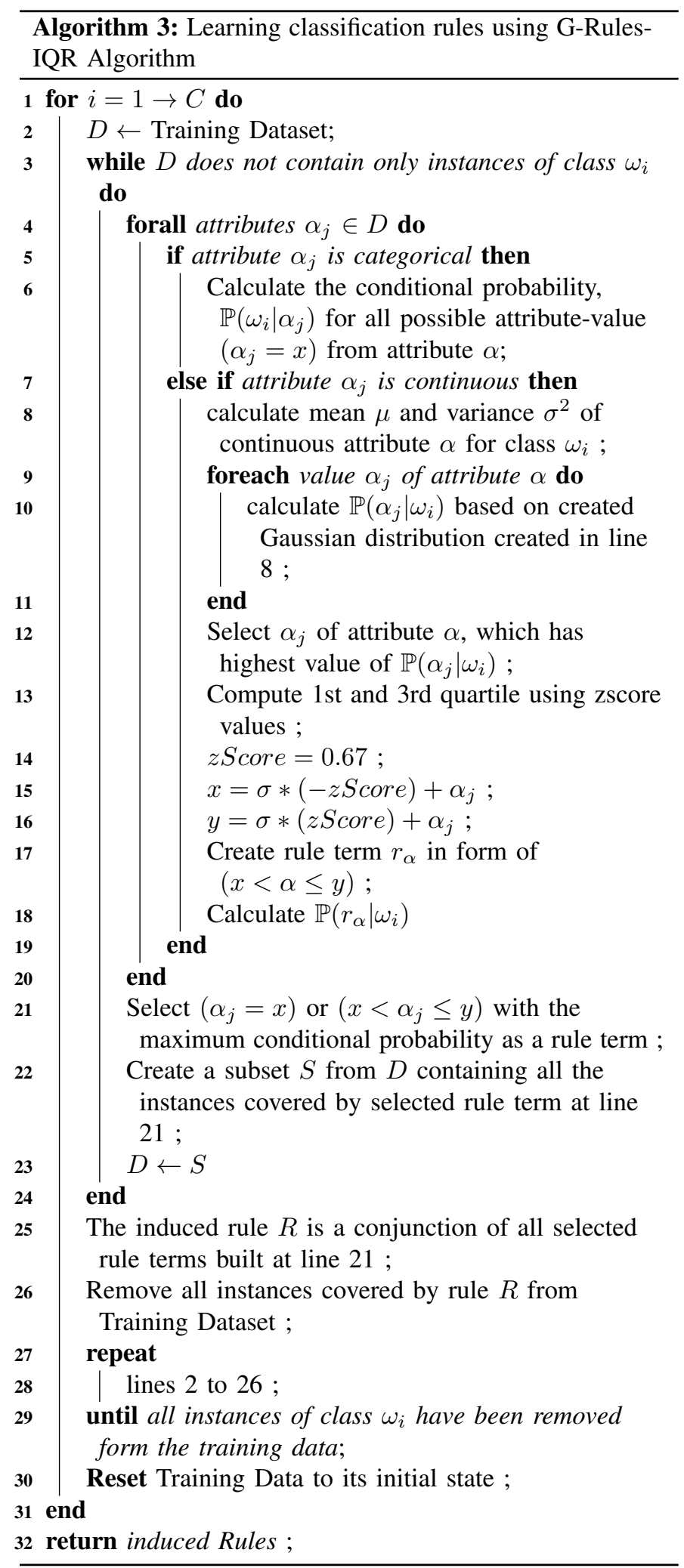

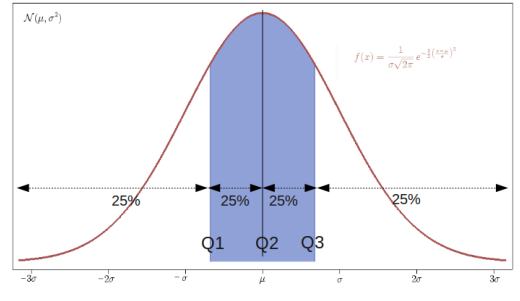

Fig. 2. Interquartile Range of Normal Random variables

used to determine the probability of a given class label $\omega_{i}$ for a valid value of attribute $\alpha_{j}$.

$$
\log \left(\mathbb{P}\left(\omega_{i} \mid \alpha_{j}\right)\right)=\log \left(\mathbb{P}\left(\alpha_{j} \mid \omega_{i}\right)\right)+\log \left(\mathbb{P}\left(\omega_{i}\right)\right)-\log \left(\mathbb{P}\left(\alpha_{j}\right)\right)
$$

The created Gaussian distribution for each class label in the training data can then be used to determine the probability of an attribute value $\alpha_{j}$ belonging to class label $\omega_{i}$, assuming that $\alpha_{j}$ lies between an upper and lower bound $\Omega_{i}$. This is based on the assumption that the values close to $\mu$ represent the most common values of continuous attribute $\alpha_{j}$ for $\omega_{i}$. G-Rules-IQR algorithm proposed in this paper makes use of the quartiles which divide the probability density function into four parts with an equal amount of data points (25\% each). As shown in Figure 2, the second quartile is identical to the median [17] while the InterQuartile Range (IQR) represents the range of attribute values that cover the middle half of the dataset. Thus, the size of coverage of this approach is dependent on the size of the datasets. We termed this G-Rules$I Q R$. In Particular, G-Rules-IQR uses the difference between the third and the first quartiles as in Equation 3 to find the upper rule term and the lower rule term boundaries. $\sigma$ is the standard deviation from the mean, $z_{1}$ is the standard score of the first quartile and is $\approx-0.67$ while $z_{3}$ is the standard score of the third quartile and is $\approx 0.67 . x$ usually represents the value of the mean $\mu$ but in case of data that is normally distributed it represents the highest probability density of value of $\mathbb{P}\left(\alpha_{j} \mid \omega_{i}\right)$ as in lines 15 and 16 of Algorithm 3. Hence, IQR can also be used as a simple test of whether or not data is normally distributed as the mean will be zero and the standard deviation will be equal to 1 [18]. The empirical results in Section IV-C show that our new improved approach G-RulesIQR can resolve most of the limitations of its predecessors described in Section II-B by improving several evaluation metrics such as accuracy, tentative accuracy, execution time and F1 Score, while not requiring the user to balance rule term boundaries.

$$
\begin{gathered}
Q_{1}=\left(\sigma * z_{1}\right)+x \\
Q_{3}=\left(\sigma * z_{3}\right)+x \\
I Q R=Q_{3}-Q_{1}
\end{gathered}
$$

\section{B. Transformation for Skewed Distribution}

A major limitation of G-Prism-FB and G-Prism-DB algorithms [13] is the assumption of normally distributed attributes. 
In order to overcome or mitigate this limitation, our proposed G-Rules-IQR algorithm incorporates a prior testing for normality for each attribute in the dataset. Hence, if values of an attribute at a particular target class are not normally distributed, then the algorithm would apply an approximate normal transformation of the attribute's values with respect to that target class. In other words, it reduces the skewness rate of attribute values from the normal distribution. A common simple transformation for a skewed long-tailed datasets is to take the logarithm of the skewed attribute values [19]. This method of transformation to normal distribution is adopted in this paper. Every attribute in a dataset prior the application of a G-Prism classifier of G-Rules-IQR, is tested for normal distribution using one of the most popular goodness-of-fit tests called Jarque-Bera test [20] and only if it is not normally distributed, then the logarithmic transformation is applied to approximate normal distribution. G-Prism algorithms are expected to have a better performance on the transformed version of the data as their rule term induction method for continuous attributes assumes normally distributed data.

\section{Comparative Experimental Evaluation}

The experiments in this study firstly aim to evaluate the performance of the new member of the Prism family (GRules-IQR) compared with its predecessors G-Prism-FB and G-Prism-DB. Unless stated otherwise the default parameters of these algorithms as stated in Section II have been used. Secondly, G-Rules-IQR and the G-Prism algorithms are compared with original Prism using three different discretisation methods to handle the continuous attributes indirectly. Further explanations about these versions of original Prism are given in Section IV-B. The implementation of G-Rules-IQR allowed to switch off the transformation to approximate normal distribution.

\section{A. Experimental Setup}

All the experiments were performed on a $2.3 \mathrm{GHz}$ Intel Core $i 7$ machine with 16GB DDR3 memory, running macOS High Sierra version 10.13.2. The evaluation procedure used in this experimental evaluation is hold-out procedure. All 18 datasets used in the experiments were picked randomly from the UCI repository [21], the only condition being that they contain continuous attributes and involve classification tasks. All algorithms have been implemented in the statistical programming language $\mathrm{R}$ [22] and re-use the same code base differing only in the methodological aspects described in this paper. The datasets have been randomly sampled without replacement into train and test datasets; whereas the test set consists of $30 \%$ the dataset and the remaining $70 \%$ was used to learn the ruleset. The datasets are described in Table I in terms of number of instances, attributes (and type of attributes) and classes. Datasets 16 and 17 contained missing values. Missing categorical values have been replaced with the most frequent categorical value for the concerning attribute, and missing continuous values have been replaced with the average value for the concerning attribute. These metrics were Number of Rules, Abstaining Rate, F1 Score, Accuracy, Tentative Accuracy and Execution Time. Please note there is a relationship between accuracy, tentative accuracy and abstaining rate. The accuracy counts abstained instances as misclassification and tentative accuracy does not include abstained instances. Therefore the higher the abstaining rate, the lower the accuracy and the higher the tentative accuracy.

TABLE I

LIST OF DATASETS USED IN THE EXPERIMENTS

\begin{tabular}{llllc}
\hline & Dataset & No. Instances & No. Attributes & No. Classes \\
\hline 1. & iris & 150 & 4 (cont) & 3 \\
2. & seeds & 210 & 7 (cont) & 3 \\
3. & wine & 178 & 13 (cont) & 3 \\
4. & blood & 748 & 5 (cont) & 2 \\
5. & bank & 1372 & 5 (cont) & 2 \\
6. & ecoli & 336 & 8 (7 cont, 1 name) & 8 \\
7. & yeast & 1484 & $9(8$ cont, 1 name) & 10 \\
8. & page & 5473 & 10 (cont) & 5 \\
9. & model & 403 & 5 (cont) & 4 \\
10. & breast & 106 & 10 (cont) & 6 \\
11. & glass & 214 & $10(9$ cont, 1 id) & 7 \\
12. & HTRU2 & 17898 & 9 (cont) & 2 \\
13. & magic & 19020 & 11 (cont) & 2 \\
14. & quality & 4898 & 12 (cont) & 11 \\
15. & letter & 20000 & 17 (cont) & 26 \\
16. & cancer & 699 & 11 (10 cont, 1 id) & 2 \\
17. & post & 90 & 9 (8 categ, 1 cont) & 3 \\
18. & EEG & 14980 & 15 (cont) & 2 \\
\hline
\end{tabular}

B. Original Prism Incorporating Different Types of Local and Global Discretisation Methods

G-Rules-IQR was compared against original Prism with different discretisation methods explained below.

1) Prism-CutP: This extended version of Prism d [8] uses binary splitting and cup-points calculations to induce rule terms from continuous attributes.

2) Prism-ChiM: ChiMerge bottom-up global discretisation method is chosen because it is a well-known approach used to deal with continuous attributes in classification tasks [23].

3) Prism-Caim: CAIM is a top-down global discretisation algorithm that does not require user defined parameters [24]. It is determined as the interdependency between the target class and the discretisation scheme of a continuous attribute.

These variations of Prism do not assume normally distributed continuous attributes, thus no transformation has been implemented for Prism using these discretisation methods.

\section{Results and Interpretation}

Tables II to VII show the results of the experiments with respect to 6 evaluation metrics. In each table the '\#' symbol refers to the number of the dataset in Table I. ' $\mathrm{T}$ ' denotes that the transformation was switched on. The best result(s) in the tables for each dataset are highlighted in bold letters.

Table II shows the results for the number of rules induced. A large number of rules may be less beneficial to the human analyst compared with a well defined smaller number of rules. This is the only metric where the original version of Prism (especially Prism-CutP) clearly outperforms G-Prism algorithms and the new G-Rules-IQR algorithm. However, 
what can also be seen is that the introduced G-Rules-IQR algorithm on the transformed data clearly outperforms its direct G-Prism predecessors (G-Prism-FB and G-Prism-DB) and in some cases even generates smaller rulesets than original Prism.

TABLE II

NUMBER OF RULES

\begin{tabular}{|c|c|c|c|c|c|c|c|c|c|}
\hline \multirow{3}{*}{ \# } & \multicolumn{3}{|c|}{ Prism } & \multicolumn{4}{|c|}{ G-Prism } & \multirow{2}{*}{\multicolumn{2}{|c|}{$\begin{array}{c}\text { G-Rules } \\
\text { IQR }\end{array}$}} \\
\hline & CutP & ChiM & Caim & & & & & & \\
\hline & & & & & $\mathrm{T}$ & & $\mathrm{T}$ & & $\mathrm{T}$ \\
\hline 1 & 12 & 8 & 9 & 9 & 10 & 20 & 21 & 18 & 18 \\
\hline 2 & 17 & 27 & 22 & 30 & 27 & 73 & 71 & 31 & 22 \\
\hline 3 & 15 & 18 & 11 & 16 & 21 & 55 & 38 & 26 & 13 \\
\hline 4 & 18 & 48 & 7 & 46 & 17 & 109 & 58 & 60 & 20 \\
\hline 5 & 14 & 196 & 8 & 176 & 250 & 466 & 483 & 101 & 89 \\
\hline 6 & 57 & 72 & 83 & 44 & 52 & 108 & 97 & 91 & 53 \\
\hline 7 & 51 & 556 & 537 & 219 & 117 & 511 & 218 & 270 & 132 \\
\hline 8 & 110 & 412 & 223 & 465 & 430 & 1236 & 1325 & 205 & 215 \\
\hline 9 & 24 & 78 & 46 & 41 & 49 & 122 & 122 & 67 & 57 \\
\hline 10 & 17 & 33 & 31 & 23 & 17 & 42 & 44 & 32 & 28 \\
\hline 11 & 56 & 63 & 64 & 45 & 40 & 75 & 81 & 67 & 30 \\
\hline 12 & 77 & 789 & 39 & 3928 & 2074 & 6292 & 7107 & 894 & 31 \\
\hline 13 & 25 & 3929 & 129 & 3133 & 4563 & 7177 & 8281 & 3467 & 155 \\
\hline 14 & 74 & 1827 & 1511 & 923 & 577 & 1576 & 1229 & 1643 & 171 \\
\hline 15 & 868 & 3801 & 3901 & 843 & 320 & 2334 & 844 & 2600 & 875 \\
\hline 16 & 33 & 41 & 37 & 23 & 6 & 48 & 9 & 49 & 11 \\
\hline 17 & 30 & 32 & 31 & 30 & 30 & 30 & 30 & 29 & 29 \\
\hline 18 & 37 & 3706 & 516 & 2602 & 4650 & 5603 & 7009 & 4585 & 4423 \\
\hline
\end{tabular}

A low abstaining rate is desired as, depending on the application, abstained instances may have to be manually classified by a human analyst. Such manual labelling can be costly. In general we can observe in Table III that in most cases the abstaining rate is low for all algorithms except for a few datasets. There is no clear winner, all G-Prism versions and G-Rules-IQR are generally competitive with the original Prism versions.

TABLE III

ABSTAINING RATE

\begin{tabular}{|c|c|c|c|c|c|c|c|c|c|}
\hline \multirow{3}{*}{ \# } & \multicolumn{3}{|c|}{ Prism } & \multicolumn{4}{|c|}{ G-Prism } & \multirow{2}{*}{\multicolumn{2}{|c|}{$\begin{array}{c}\text { G-Rules } \\
\text { IQR }\end{array}$}} \\
\hline & CutP & ChiM & Caim & \multicolumn{2}{|c|}{ DB } & \multicolumn{2}{|c|}{ FB } & & \\
\hline & & & & & $\mathrm{T}$ & & $\mathrm{T}$ & & $\mathrm{T}$ \\
\hline 1 & 0.09 & 0.00 & 0.04 & 0.04 & 0.02 & 0.02 & 0.02 & 0.07 & 0.07 \\
\hline 2 & 0.17 & 0.05 & 0.03 & 0.10 & 0.10 & 0.06 & 0.05 & 0.03 & 0.03 \\
\hline 3 & 0.17 & 0.11 & 0.08 & 0.11 & 0.04 & 0.19 & 0.04 & 0.17 & 0.06 \\
\hline 4 & 0.00 & 0.02 & 0.00 & 0.10 & 0.02 & 0.23 & 0.00 & 0.08 & 0.00 \\
\hline 5 & 0.00 & 0.10 & 0.00 & 0.08 & 0.13 & 0.09 & 0.09 & 0.00 & 0.02 \\
\hline 6 & 0.14 & 0.11 & 0.12 & 0.24 & 0.10 & 0.31 & 0.16 & 0.20 & 0.08 \\
\hline 7 & 0.07 & 0.17 & 0.16 & 0.36 & 0.08 & 0.45 & 0.08 & 0.36 & 0.07 \\
\hline 8 & 0.01 & 0.05 & 0.01 & 0.03 & 0.03 & 0.03 & 0.03 & 0.04 & 0.02 \\
\hline 9 & 0.50 & 0.26 & 0.03 & 0.17 & 0.12 & 0.28 & 0.28 & 0.19 & 0.30 \\
\hline 10 & 0.31 & 0.06 & 0.06 & 0.31 & 0.19 & 0.44 & 0.22 & 0.19 & 0.19 \\
\hline 11 & 0.11 & 0.08 & 0.20 & 0.54 & 0.12 & 0.46 & 0.14 & 0.42 & 0.11 \\
\hline 12 & 0.00 & 0.03 & 0.00 & 0.02 & 0.00 & 0.03 & 0.00 & 0.01 & 0.00 \\
\hline 13 & 0.00 & 0.23 & 0.00 & 0.31 & 0.00 & 0.34 & 0.01 & 0.16 & 0.00 \\
\hline 14 & 0.00 & 0.22 & 0.12 & 0.40 & 0.01 & 0.35 & 0.02 & 0.41 & 0.01 \\
\hline 15 & 0.38 & 0.13 & 0.14 & 0.16 & 0.01 & 0.16 & 0.03 & 0.15 & 0.04 \\
\hline 16 & 0.02 & 0.01 & 0.00 & 0.03 & 0.00 & 0.02 & 0.02 & 0.02 & 0.00 \\
\hline 17 & 0.04 & 0.04 & 0.11 & 0.04 & 0.04 & 0.04 & 0.04 & 0.11 & 0.11 \\
\hline 18 & 0.00 & 0.16 & 0.01 & 0.35 & 0.15 & 0.33 & 0.12 & 0.23 & 0.19 \\
\hline
\end{tabular}

Table IV lists the results for the F1 Score for each of the classifiers. This is the harmonic mean of precision and recall. In multi-class problems such as in these datasets, precision and recall are computed by building an average of these metrics' values for each class. The results show that the proposed method G-Rules-IQR with transformation achieved the best
F1 Score on 11 out of 18 datasets. That is more often than any of the other evaluated algorithms. For most of the 7 datasets where the G-Rules-IQR with transformation did not achieve the best F1 Score, it was still close to the best performing F1 Score, in particular for 3 datasets it achieved an F1 Score that was at most only 3\% lower than the best F1 Score. This shows that the method is competitive and in some cases even outperforms its competitors.

TABLE IV

F1 SCORE

\begin{tabular}{ccccccccccc}
\hline & \multicolumn{3}{c}{ Prism } & \multicolumn{4}{c}{ G-Prism } & \multicolumn{3}{c}{ G-Rules } \\
\cline { 2 - 11 }$\#$ & CutP & ChiM & Caim & \multicolumn{2}{c}{ DB } & \multicolumn{3}{c}{ FB } & \multicolumn{2}{c}{ IQR } \\
\cline { 2 - 11 } & & & & \multicolumn{3}{c}{ T } & & T & & T \\
\hline 1 & 0.93 & 0.91 & 0.95 & 0.93 & 0.91 & 0.93 & 0.93 & $\mathbf{0 . 9 6}$ & $\mathbf{0 . 9 6}$ \\
2 & 0.96 & 0.97 & 0.95 & 0.93 & $\mathbf{1 . 0 0}$ & 0.89 & $\mathbf{1 . 0 0}$ & 0.94 & $\mathbf{1 . 0 0}$ \\
3 & $\mathbf{0 . 9 8}$ & 0.93 & 0.92 & 0.96 & $\mathbf{0 . 9 8}$ & $\mathbf{0 . 9 8}$ & $\mathbf{0 . 9 8}$ & 0.89 & $\mathbf{0 . 9 8}$ \\
4 & 0.87 & 0.87 & 0.87 & 0.89 & $\mathbf{1 . 0 0}$ & 0.90 & $\mathbf{1 . 0 0}$ & 0.89 & 0.98 \\
5 & 0.80 & $\mathbf{0 . 9 9}$ & 0.94 & 0.96 & 0.97 & 0.96 & 0.97 & 0.98 & $\mathbf{0 . 9 9}$ \\
6 & 0.77 & 0.61 & 0.71 & 0.72 & $\mathbf{0 . 8 0}$ & 0.72 & 0.61 & 0.62 & 0.79 \\
7 & 0.33 & 0.53 & 0.55 & 0.49 & 0.75 & 0.49 & 0.81 & 0.54 & $\mathbf{0 . 8 6}$ \\
8 & 0.64 & 0.74 & 0.78 & 0.80 & 0.84 & 0.82 & 0.85 & 0.89 & $\mathbf{0 . 9 3}$ \\
9 & 0.82 & 0.91 & 0.87 & 0.92 & 0.86 & 0.84 & 0.84 & 0.94 & $\mathbf{0 . 9 6}$ \\
10 & 0.81 & 0.73 & 0.83 & $\mathbf{0 . 9 3}$ & 0.93 & 0.79 & 0.77 & 0.80 & 0.81 \\
11 & 0.64 & 0.73 & 0.84 & 0.67 & $\mathbf{0 . 9 7}$ & 0.44 & 0.90 & 0.61 & 0.86 \\
12 & 0.96 & 0.99 & 0.99 & 0.99 & $\mathbf{1 . 0 0}$ & 0.99 & $\mathbf{1 . 0 0}$ & 0.99 & $\mathbf{1 . 0 0}$ \\
13 & 0.80 & 0.98 & 0.85 & 0.88 & 0.95 & 0.87 & 0.95 & 0.91 & $\mathbf{1 . 0 0}$ \\
14 & 0.29 & 0.49 & 0.35 & 0.50 & $\mathbf{0 . 9 5}$ & 0.50 & 0.79 & 0.55 & 0.79 \\
15 & 0.90 & 0.82 & 0.83 & 0.87 & $\mathbf{0 . 9 9}$ & 0.88 & $\mathbf{0 . 9 9}$ & 0.88 & $\mathbf{0 . 9 9}$ \\
16 & 0.97 & 0.97 & 0.97 & 0.97 & $\mathbf{1 . 0 0}$ & 0.98 & $\mathbf{1 . 0 0}$ & 0.98 & $\mathbf{1 . 0 0}$ \\
17 & 0.38 & 0.53 & $\mathbf{0 . 6 9}$ & 0.49 & 0.49 & 0.49 & 0.49 & 0.52 & 0.52 \\
18 & 0.71 & 0.83 & 0.76 & 0.79 & 0.79 & 0.77 & 0.78 & $\mathbf{0 . 8 7}$ & 0.86 \\
\hline
\end{tabular}

TABLE V

ACCURACY

\begin{tabular}{|c|c|c|c|c|c|c|c|c|c|}
\hline \multirow{3}{*}{ \# } & \multicolumn{3}{|c|}{ Prism } & \multicolumn{4}{|c|}{ G-Prism } & \multirow{2}{*}{\multicolumn{2}{|c|}{$\begin{array}{c}\text { G-Rules } \\
\text { IQR }\end{array}$}} \\
\hline & CutP & ChiM & Caim & & & & & & \\
\hline & & & & & $\mathrm{T}$ & & $\mathrm{T}$ & & $\mathrm{T}$ \\
\hline 1 & 0.87 & 0.91 & 0.91 & 0.91 & 0.91 & 0.91 & 0.91 & 0.91 & 0.91 \\
\hline 2 & 0.92 & 0.95 & 0.94 & 0.86 & 0.90 & 0.87 & 0.95 & 0.87 & 0.97 \\
\hline 3 & 0.89 & 0.83 & 0.85 & 0.89 & 0.96 & 0.79 & 0.94 & 0.85 & 0.94 \\
\hline 4 & 0.76 & 0.77 & 0.77 & 0.77 & 0.98 & 0.77 & 1.00 & 0.77 & 0.97 \\
\hline 5 & 0.72 & 0.93 & 0.94 & 0.92 & 0.92 & 0.90 & 0.93 & 0.98 & 0.98 \\
\hline 6 & 0.77 & 0.75 & 0.75 & 0.72 & 0.85 & 0.65 & 0.77 & 0.73 & 0.91 \\
\hline 7 & 0.37 & 0.51 & 0.51 & 0.44 & 0.87 & 0.46 & 0.89 & 0.49 & 0.89 \\
\hline 8 & 0.95 & 0.95 & 0.96 & 0.95 & 0.97 & 0.95 & 0.97 & 0.96 & 0.98 \\
\hline 9 & 0.61 & 0.74 & 0.83 & 0.78 & 0.76 & 0.66 & 0.66 & 0.82 & 0.72 \\
\hline 10 & 0.59 & 0.72 & 0.81 & 0.66 & 0.78 & 0.50 & 0.69 & 0.66 & 0.66 \\
\hline 11 & 0.60 & 0.77 & 0.75 & 0.55 & 0.86 & 0.51 & 0.83 & 0.58 & 0.86 \\
\hline 12 & 0.92 & 0.97 & 0.98 & 0.97 & 1.00 & 0.96 & 0.99 & 0.98 & 1.00 \\
\hline 13 & 0.67 & 0.86 & 0.80 & 0.74 & 0.94 & 0.72 & 0.93 & 0.80 & 1.00 \\
\hline 14 & 0.49 & 0.63 & 0.58 & 0.56 & 0.98 & 0.59 & 0.97 & 0.60 & 0.99 \\
\hline 15 & 0.57 & 0.72 & 0.71 & 0.73 & 0.98 & 0.75 & 0.96 & 0.75 & 0.96 \\
\hline 16 & 0.96 & 0.95 & 0.96 & 0.94 & 1.00 & 0.96 & 1.00 & 0.96 & 1.00 \\
\hline 17 & 0.59 & 0.74 & 0.74 & 0.63 & 0.63 & 0.63 & 0.63 & 0.67 & 0.67 \\
\hline 18 & 0.56 & 0.75 & 0.70 & 0.67 & 0.71 & 0.66 & 0.72 & 0.77 & 0.77 \\
\hline
\end{tabular}

Table V lists the results for the accuracy for each of the classifiers and abstained instances are counted as misclassifications. G-Rules-IQR with transformation achieved the best accuracy on 12 datasets, more often than any of the other evaluated algorithms. On 3 datasets G-Rules-IQR was not the best method, but was still very close within $3 \%$ of the best accuracy. Only on 3 datasets $(9,10$ and 17) G-Rules-IQR's accuracy was much lower than the other evaluated algorithms. However, these datasets also cause a relatively high abstaining rate. 
Table VI lists the results for the tentative accuracy. In most cases the proposed method G-Rules-IQR with transformation achieved the highest tentative accuracy. In particular it achieved the highest tentative accuracy on 13 out of 18 datasets and on 3 out the the remaining 5 datasets its accuracy was within $3 \%$ of the best accuracy.

TABLE VI

TEntative ACCURACy

\begin{tabular}{lllllccccc}
\hline & \multicolumn{3}{c}{ Prism } & \multicolumn{4}{c}{ G-Prism } & \multicolumn{2}{c}{ G-Rules } \\
\cline { 2 - 10 }$\#$ & CutP & ChiM & Caim & \multicolumn{2}{c}{ DB } & \multicolumn{2}{c}{ FB } & \multicolumn{2}{c}{ IQR } \\
\cline { 2 - 11 } & & & & \multicolumn{3}{c}{ T } & T & & T \\
\hline 1 & 0.93 & 0.91 & $\mathbf{0 . 9 5}$ & 0.93 & 0.91 & 0.93 & 0.93 & $\mathbf{0 . 9 5}$ & $\mathbf{0 . 9 5}$ \\
3 & 0.96 & 0.97 & 0.95 & 0.93 & $\mathbf{1 . 0 0}$ & 0.93 & $\mathbf{1 . 0 0}$ & 0.89 & $\mathbf{1 . 0 0}$ \\
4 & $\mathbf{0 . 9 8}$ & 0.94 & 0.92 & 0.96 & $\mathbf{0 . 9 8}$ & 0.88 & $\mathbf{0 . 9 8}$ & $\mathbf{0 . 9 8}$ & $\mathbf{0 . 9 8}$ \\
5 & 0.76 & 0.78 & 0.77 & 0.81 & $\mathbf{1 . 0 0}$ & 0.82 & $\mathbf{1 . 0 0}$ & 0.80 & 0.97 \\
6 & 0.72 & $\mathbf{0 . 9 9}$ & 0.94 & 0.96 & 0.97 & 0.95 & 0.97 & 0.98 & $\mathbf{0 . 9 9}$ \\
7 & 0.86 & 0.81 & 0.83 & 0.86 & 0.91 & 0.86 & 0.86 & 0.84 & $\mathbf{0 . 9 4}$ \\
8 & 0.95 & 0.54 & 0.57 & 0.54 & 0.95 & 0.58 & 0.96 & 0.59 & $\mathbf{0 . 9 7}$ \\
9 & 0.97 & 0.97 & 0.96 & 0.98 & 0.96 & 0.97 & 0.98 & $\mathbf{0 . 9 9}$ \\
10 & 0.86 & 0.91 & 0.86 & 0.91 & 0.85 & 0.83 & 0.83 & 0.94 & $\mathbf{0 . 9 5}$ \\
11 & 0.64 & 0.77 & 0.87 & $\mathbf{0 . 9 5}$ & 0.92 & 0.89 & 0.84 & 0.81 & 0.81 \\
12 & 0.92 & 0.99 & 0.98 & 0.98 & $\mathbf{1 . 0 0}$ & 0.98 & 0.99 & 0.98 & $\mathbf{1 . 0 0}$ \\
13 & 0.67 & 0.97 & 0.80 & 0.82 & 0.94 & 0.80 & 0.93 & 0.87 & $\mathbf{1 . 0 0}$ \\
14 & 0.49 & 0.66 & 0.61 & 0.63 & 0.99 & 0.66 & 0.98 & 0.69 & $\mathbf{1 . 0 0}$ \\
15 & 0.90 & 0.82 & 0.82 & 0.87 & $\mathbf{0 . 9 9}$ & 0.88 & $\mathbf{0 . 9 9}$ & 0.88 & $\mathbf{0 . 9 9}$ \\
16 & 0.97 & 0.96 & 0.96 & 0.97 & $\mathbf{1 . 0 0}$ & 0.97 & $\mathbf{1 . 0 0}$ & 0.98 & $\mathbf{1 . 0 0}$ \\
17 & 0.62 & 0.73 & $\mathbf{0 . 7 5}$ & 0.65 & 0.65 & 0.65 & 0.65 & 0.67 & 0.67 \\
18 & 0.56 & 0.79 & 0.71 & 0.74 & 0.73 & 0.72 & 0.72 & $\mathbf{0 . 8 4}$ & 0.83 \\
\hline
\end{tabular}

Table VII lists the results for the execution times. These also include the time needed approximating normal distribution for G-Prism classifiers and G-Rules-IQR with transformation. In most cases the proposed method (G-Rules-IQR with transformation) achieved shortest execution times. On 15 out of 18 datasets it was the fastest algorithm and thus clearly outperforms its competitors. Overall the time complexity of G-Rules-IQR is expected to be similar to that of G-Prism and Prism algorithms with respect to the number of instances $N$ and number of attributes $M$. However, it is expected that GRules-IQR is faster. In [25] the authors estimated the worst case time complexity of a Prism classifier to be approximately $O\left(N^{2} M\right)$. In the worst case each rule covers exactly one data instance and each rule has two rule terms per attribute. This is a very unlikely case and time complexity is strongly dependent on the pattern in the data that can be expressed in the form of rules. The worst case of G-Rules-IQR and GPrism classifiers would induce only 1 rule term per attribute and thus already divides the worst case complexity by 2 . In addition G-Rules-IQR is expected to be faster than G-PrismDB and Prism because of the number of calculations required to induce a rule term. In the worst case scenario Prism and G-Prism have to evaluate either several cut-point calculations or rule term boundaries, whereas G-Rules-IQR only has to calculate the quartiles. Also G-Rules-IQR with transformation has a lower runtime than G-Rules-IQR even though there is an additional operation. However, this is likely because G-RulesIQR with transformation produces fewer rules than G-RulesIQR without transformation in most cases.

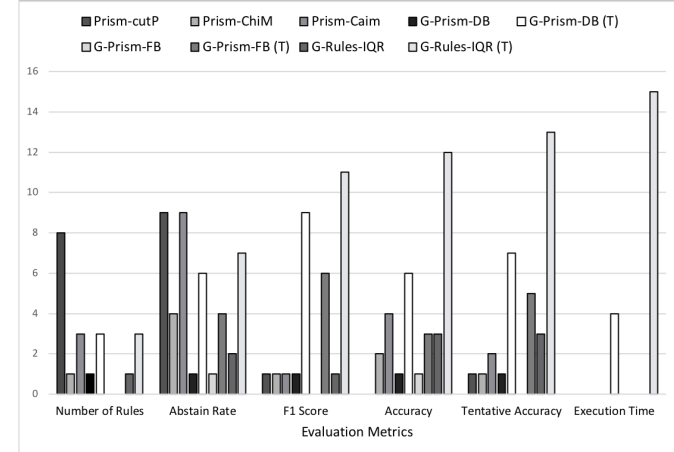

Fig. 3. Summary of results

\section{Summary of Results}

Figure 3 summarises how often a particular algorithm outperformed all of its competitors relating to the evaluation measures observed in Section IV-C. It can be seen that the proposed G-Rules-IQR algorithm with transformation outperformed its competitors in terms of F1 Score, accuracy, tentative accuracy and execution time.

\section{CONCLUSION}

The paper presents the rule-based G-Rules-IQR algorithm for continuous attributes, a new member of the Prism family of predictive rule induction algorithms. Previous work in this area (G-Prism-FB and G-Prism-DB) addressed the shortcoming of binary splitting to induce rule terms, which leads to rule terms covering irrelevant proportions of the training data. G-PrismFB and G-Prism-DB generate numeric expressive rule terms using GPDD. There are 4 limitations of these methods. These are (1) more accurate G-Prism-DB has a longer execution time than G-Prism-FB, (2) both algorithms have a higher abstaining rate than the original Prism classifier, (3) G-Prism-DB requires user defined rule term boundary thresholds and (4) both approaches assume normally distributed continuous attributes. With respect to the assumption of normally distributed data, an approximation towards normally distributed data is integrated into the G-Rules-IQR algorithm. With respect to underfitting rule term boundaries, the method optimises these boundaries by removing user defined thresholds through using IQR. The approach was termed G-Rules-IQR with transformation and was evaluated empirically and comparatively with its predecessors including Prism with binary splitting and Prism with various well established global discretisation methods. Overall G-Rules-IQR with transformation outperformed its competitors with respect to F1 Score, accuracy, tentative accuracy and execution time. With regards to limitation (1), G-RulesIQR achieves shorter execution times than its competitors, with respect to limitation (2) G-Prism achieves a competitive (similar) abstaining rate as its competitors, with respect to limitation (3) G-Rules-IQR does not require user input for rule term boundary thresholds and with respect to limitation (4) the normal distribution approximation made G-Rules-IQR the best performing Prism based classifier in this paper. 
TABLE VII

EXECUTION TIME

\begin{tabular}{|c|c|c|c|c|c|c|c|c|c|}
\hline \multirow{3}{*}{ \# } & \multicolumn{3}{|c|}{ Prism } & \multicolumn{4}{|c|}{ G-Prism } & \multirow{2}{*}{\multicolumn{2}{|c|}{$\begin{array}{c}\text { G-Rules } \\
\text { IQR }\end{array}$}} \\
\hline & CutP & ChiM & Caim & & & & & & \\
\hline & & & & & $\mathrm{T}$ & & $\mathrm{T}$ & & $\mathrm{T}$ \\
\hline 1 & 2.66 & 2.18 & 2.39 & 1.92 & 1.65 & 2.98 & 2.61 & 1.95 & 1.65 \\
\hline 2 & 3.86 & 12.11 & 8.10 & 5.97 & 5.52 & 4.46 & 4.17 & 3.07 & 2.63 \\
\hline 3 & 4.93 & 11.27 & 8.52 & 8.09 & 5.86 & 4.73 & 4.11 & 3.93 & 2.75 \\
\hline 4 & 6.10 & 15.44 & 7.86 & 12.22 & 5.33 & 11.20 & 6.90 & 8.33 & 5.33 \\
\hline 5 & 15.07 & 224.80 & 25.31 & 32.70 & 31.64 & 38.50 & 38.78 & 15.64 & 13.48 \\
\hline 6 & 10.76 & 18.14 & 22.62 & 9.69 & 6.58 & 8.37 & 6.54 & 7.89 & 5.23 \\
\hline 7 & 40.37 & 262.30 & 394.20 & 98.40 & 26.08 & 100.20 & 30.32 & 76.20 & 23.60 \\
\hline 8 & 1068.00 & 546.00 & 407.40 & 295.80 & 256.80 & 440.40 & 417.00 & 115.20 & 87.00 \\
\hline 9 & 12.29 & 21.77 & 12.49 & 7.78 & 7.46 & 6.51 & 6.25 & 4.98 & 4.25 \\
\hline 10 & 24.37 & 19.60 & 13.77 & 6.78 & 4.55 & 3.11 & 3.12 & 3.50 & 2.88 \\
\hline 11 & 39.87 & 18.62 & 27.06 & 9.26 & 5.41 & 6.44 & 4.63 & 7.37 & 3.18 \\
\hline 12 & 352.80 & 65520.00 & 2492.40 & 6600.00 & 3066.00 & 5652.00 & 7200.00 & 1055.00 & 165.60 \\
\hline 13 & 568.80 & 234144.00 & 3321.00 & 6500.00 & 5184.00 & 6768.00 & 6840.00 & 6372.00 & 238.80 \\
\hline 14 & 198.00 & 1622.00 & 2428.20 & 509.40 & 248.40 & 459.60 & 330.60 & 1079.00 & 86.40 \\
\hline 15 & 1720.00 & 10800.00 & 11844.00 & 6000.00 & 754.80 & 2583.00 & 939.00 & 3182.00 & 1049.00 \\
\hline 16 & 11.94 & 12.82 & 13.72 & 11.97 & 5.48 & 9.04 & 5.76 & 8.90 & 5.03 \\
\hline 17 & 3.66 & 28.22 & 5.88 & 4.28 & 3.20 & 3.64 & 3.50 & 4.36 & 3.53 \\
\hline 18 & 262.20 & 8028.00 & 1752.00 & 6540.00 & 5472.00 & 4464.00 & 5508.00 & 10224.00 & 10296.00 \\
\hline
\end{tabular}

Ongoing work comprises a voting strategy for G-RulesIQR rulesets. Currently the first rule that fires produces the prediction, however, the rule order has no relationship to the individual rule's accuracy. Thus a rule filter and weighting mechanism (according to rule quality) is currently being investigated. Also the fact that currently G-Rules-IQR does not take attribute dependencies into consideration is currently being investigated (see limitation 5 in Section II-B). For example, the expressiveness of the ruleset could be improved by not allowing rule terms of coexisting attributes in the same rule, which will lead to shorter rules and thus smaller rulesets.

\section{REFERENCES}

[1] J. Han, J. Pei, and M. Kamber, Data mining: concepts and techniques. Elsevier, 2011.

[2] K. Grbczewski, "Techniques of decision tree induction," in MetaLearning in Decision Tree Induction. Springer, 2014, pp. 11-117.

[3] J. R. Quinlan, C4. 5: programs for machine learning. Elsevier, 2014.

[4] - "Generating production rules from decision trees," vol. 87, pp. 304-307, 1987.

[5] I. H. Witten, E. Frank, M. A. Hall, and C. J. Pal, Data Mining: Practical machine learning tools and techniques. Morgan Kaufmann, 2016.

[6] J. Cendrowska, "Prism: An algorithm for inducing modular rules," International Journal of Man-Machine Studies, vol. 27, no. 4, pp. 349370, 1987.

[7] F. Stahl and M. Bramer, "Computationally efficient induction of classification rules with the pmcri and j-pmcri frameworks," Knowledge-Based Systems, vol. 35, pp. 49-63, 2012.

[8] M. Bramer, Principles of data mining. Springer, 2007, vol. 180.

[9] W. W. Cohen, "Fast effective rule induction," in Machine Learning Proceedings 1995. Elsevier, 1995, pp. 115-123.

[10] P. Clark and T. Niblett, "The cn2 induction algorithm," Machine learning, vol. 3, no. 4, pp. 261-283, 1989.

[11] J. Fürnkranz, D. Gamberger, and N. Lavrač, Foundations of rule learning. Springer Science \& Business Media, 2012.

[12] M. Bramer, "An information-theoretic approach to the pre-pruning of classification rules," in International Conference on Intelligent Information Processing. Springer, 2002, pp. 201-212.

[13] M. Almutairi, F. Stahl, and M. Bramer, "Improving modular classification rule induction with g-prism using dynamic rule term boundaries," in International Conference on Innovative Techniques and Applications of Artificial Intelligence. Springer, 2017, pp. 115-128.

[14] M. Almutairi, F. Stahl, M. Jennings, T. Le, and M. Bramer, "Towards expressive modular rule induction for numerical attributes," in Research and Development in Intelligent Systems XXXIII: Incorporating Applications and Innovations in Intelligent Systems XXIV. Springer, 2016, pp. 229-235.

[15] M. Bramer, "Automatic induction of classification rules from examples using n-prism," in Research and development in intelligent systems XVI. Springer, 2000, pp. 99-121.

[16] T. Le, F. Stahl, J. B. Gomes, M. M. Gaber, and G. Di Fatta, "Computationally efficient rule-based classification for continuous streaming data," in Research and Development in Intelligent Systems XXXI. Springer, 2014, pp. 21-34.

[17] C. Walck, "Hand-book on statistical distributions for experimentalists," Tech. Rep., 1996.

[18] L. Statistics. [Online]. Available: https://statistics.laerd.com

[19] H. C. Thode, Testing for normality. CRC press, 2002, vol. 164.

[20] C. M. Jarque and A. K. Bera, "Efficient tests for normality, homoscedasticity and serial independence of regression residuals," Economics letters, vol. 6, no. 3, pp. 255-259, 1980.

[21] D. N. A. Asuncion, "UCI machine learning repository," 2007. [Online]. Available: http://www.ics.uci.edu/ mlearn/MLRepository.html

[22] R Development Core Team, R: A Language and Environment for Statistical Computing, R Foundation for Statistical Computing, Vienna, Austria, 2008, ISBN 3-900051-07-0. [Online]. Available: http://www.R-project.org

[23] R. Kerber, "Chimerge: Discretization of numeric attributes," in Proceedings of the tenth national conference on Artificial intelligence. Aaai Press, 1992, pp. 123-128.

[24] L. A. Kurgan and K. J. Cios, "Caim discretization algorithm," IEEE transactions on Knowledge and Data Engineering, vol. 16, no. 2, pp. 145-153, 2004.

[25] F. Stahl, D. May, H. Mills, M. Bramer, and M. M. Gaber, "A scalable expressive ensemble learning using random prism: a mapreduce approach," Transactions on Large-Scale Data- and Knowledge-Centered Systems, vol. 9070, pp. 90-107, 2015. 